\title{
Source Zone Characterization Tool for Contaminated Groundwater Aquifers
}

\author{
Mohamed Mostafa Mohamed
}

\begin{abstract}
Knowledge of contaminants source zones characteristics is an important initial step in the development of conceptual site model which is necessary to make informed decisions about trigger levels for restoration. The recently developed dipole flow and reactive tracer test (DFRTT) is used to justify usefulness of monitored natural attenuation of contaminated aquifers. It uses the dipole flow test which circulates groundwater between isolated injection and extraction chambers within a single borehole to delineate heterogeneous hydraulic properties. The aim of this paper is to extend this approach by modifying the developed DFRTT into a multiple flow and reactive tracer test (MFRTT) to facilitate contaminant source zone characterization. These modifications maximize the usefulness of the DFRTT to attain detailed description of the physical, chemical and biological properties of contaminants source zones. Mathematical and numerical models that examine the theoretical feasibility of the MFRTT are the main objectives of this paper.
\end{abstract}

Index Terms-Bioremediation, groundwater contamination, source zone.

\section{INTRODUCTION}

Source zone characterization has become an important initial step for the in situ treatment of contaminated subsurface environments. This technology requires a high degree of certainty in the conceptual site model and the underlying reactive transport processes. In order to gather this site specific information, site characterization studies must be performed; however, the expense of these studies detract from the low-cost nature of monitored natural attenuation (MNA). Hence cost-effective site characterization techniques are required which provide a level of information suitable for demonstrating that in situ processes are present for MNA to be a viable treatment option. In an attempt to develop a dependable low-cost site characterization tool, the Groundwater Protection and Restoration Group at the University of Sheffield, UK, extended the dipole-flow test, developed by Kabala [1], to include reactive tracers so that in situ reactive parameters required to support MNA can be estimated. This site characterization test was called The Dipole Flow Reactive Tracer Test (DFRTT) [2]-[4].

In the late 1980s, hydrogeologist and engineers applied vertically circulating flow fields (dipole flow field) as a means to remediate contaminated ground water by injecting into one interval and extracting from another interval of a single well.

Manuscript received August 11, 2014; revised January 20, 2015. This work was supported in part by the UAEU.

Mohamed Mostafa Mohamed is with the Civil and Environmental Engineering Department, U.A.E. University, Al-Ain, 15551, UAE (e-mail: m.mohamed@uaeu.ac.ae).
The extracted water was either treated by air stripping within the well, or amendments (e.g., oxygen, nutrients, or chemicals) were added to the extracted water before it was re-injected. Herrling and Stamm [5] were the first to investigate the general features of vertically circulating flow fields in support of their use as a remedial technology. A three-dimensional numerical model that simulated the radially symmetric flow field in a confined aquifer was used to estimate the basic flow field, and the effects of an ambient flow field were superimposed on the resulting velocity field. A simple particle tracking scheme was used to estimate capture zones. This modeling approach was used to generate a host of relationships to aid in the design of such remedial systems.

Also with a focus on vertical circulation wells and their capture zones, Philip and Walter [6] developed an analytical method for simulating the flow field and fluid path lines in a homogeneous anisotropic confined aquifer with a regional gradient. They used the hydraulic head change due to a point sink in an infinite homogeneous porous medium, the principle of superposition to develop a line sink/source, the method of images to satisfy the confined aquifer boundary conditions, and spatial scaling to account for the effects of an anisotropic hydraulic conductivity field. This analytical tool was used to investigate the capture zone properties for various well configurations, lengths of well screens, and changes in hydraulic conductivity ratios.

Kabala [1] was the first to propose the dipole flow test (DFT) as a technique to characterize the horizontal and vertical hydraulic conductivity, and the specific storativity of aquifer material. His mathematical interpretation model assumed: (i) a fully penetrating well, (ii) a homogeneous radially symmetric anisotropic leaky or confined aquifer of infinite extent, (iii) no well bore storage, (iv) no skin effect, (v) no storage in the confining layer, and (vi) no well losses. With these assumptions he used the principle of superposition describing the drawdown in a partially penetrating observation well.

Extending from the effort of Kabala [1], Zlotnik and Ledder [7] developed a number of mathematical models with a focus on understanding the kinematic flow structure around the dipole device, and the drawdown in the well chambers in a uniform anisotropic infinite aquifer. They investigated the region of influence by treating the injection/extraction chambers as point source/sinks and using a Taylor series expansion of the solution of the total drawdown. They also considered the drawdown produced by treating the injection/extraction chambers as a linear source/sink rather than as a point source/sink again for an infinite aquifer. A practical outcome from this investigation was equations for 
the steady-state Darcy velocity field as a function of the spatial coordinates $(r, z)$. Using these velocity equations along with the definition of Stokes' stream function for asymmetric flow, they derived an expression for Stokes' stream function as a function of $(r, z)$. This expression allows for the direct calculation of the path-lines in a dipole flow field (under the stated assumptions).

Zlotnik and Zurbuchen [8] presented construction details of a dipole system and related components, and provided guidelines for the interpretation of pseudo steady-state field data to estimate hydraulic conductivity. Their interpretation method involves an assumption of steady-state flow and a prior knowledge of the aquifer isotropy ratio $\left(a^{2}=K_{r} / K_{z}\right)$. They reported on the results from 153 DFTs conducted in a single well varying the flow rate, and the length of the injection and extraction chambers. In general, the results showed that, for the site investigated, all dipole configurations produced the same hydraulic conductivity profile and compared well with the magnitude and trend produced from grain-size analysis estimates (using the Hazen formula).

Zlotnik et al. [9] compared hydraulic conductivity estimates from the DFT conducted in 14 boreholes at the Horkheimer Insel field site in Germany to hydraulic conductivity estimated from sieve analysis, constant head permeameter, flow meter, and pump tests. Their conclusions were that the DFT hydraulic conductivity estimates were comparable to the borehole flow-meter results, but overall results from the DFT should be treated as another hydraulic testing technique due in part to the scale issue (spatial scale of $\sim 1 \mathrm{~m}$ ).

Halihan and Zlotnik [10] presented a modification of the DFT in which the injection and extraction chambers are different lengths and termed this the asymmetric DFT. The testing procedures for an asymmetric DFT are essentially the same as for the symmetric DFT; however, the interpretation method needs to be modified to account for the different chamber lengths. Halihan and Zlotnik [10] modified the approach used by Zlotnik and Ledder [7] to produce expressions for the horizontal hydraulic conductivity near the upper and lower chambers. These expressions are functions of the flow rate, steady-state drawdown in each chamber, the chamber length, and shape factor. They used the asymmetric DFT and associated interpretation approach to estimate the horizontal hydraulic conductivity of a fractured dolomite aquifer at the Bissen Quarry site in Wisconsin, USA. The estimated vertical hydraulic conductivity profile from the asymmetric DFTs was similar in order of magnitude to those estimated by the conventional double packer slug extraction tests.

\section{GOVERNING EQUATIONS}

\section{A. Flow Equations}

Analytical solutions have been presented by Zlotnik and Ledder [7] to design and interpret a dipole flow test in a homogeneous porous media. Horizontal and vertical hydraulic conductivities, the flow field and the volume of aquifer sampled can be evaluated from the analytical expressions given.
In order to account for heterogeneous layered formations and include different boundary conditions encountered for real field experiments, the groundwater flow equation is solved numerically to compute the flow field. The stream function can be evaluated from the equation below for a radial symmetric configuration.

$$
\frac{1}{r} \frac{\partial}{\partial r}\left(\frac{1}{K_{z}} r \frac{\partial \psi}{\partial r}\right)+\frac{\partial}{\partial z}\left(\frac{1}{K_{r}} \frac{\partial \psi}{\partial z}\right)=0
$$

The following boundary conditions are used:

1) within the injection and extraction chambers: $\psi \in[0,1]$

2) between the extraction chambers on the lhs: $\psi=0$

3) on the top, bottom and rhs: $\psi=1$

The pseudo-potential functions should be solved to generate an orthogonal flow net. For homogeneous and isotropic systems, the head potential function is orthogonal to the stream function. However for heterogeneous and anisotropic systems, only the pseudo-potential function $\phi$ is orthogonal to the stream function $\psi$. It can be evaluated by solving the following equation:

$$
\frac{1}{r} \frac{\partial}{\partial r}\left(-K_{\phi} r \frac{\partial \phi}{\partial r}\right)+\frac{\partial}{\partial z}\left(-K_{\phi} \frac{\partial \phi}{\partial \mathrm{z}}\right)=0
$$

where the pseudo-potential conductivity is given by:

$$
K_{\varphi}=\frac{\left(\frac{\partial \psi}{\partial r}\right)^{2}+\left(\frac{\partial \psi}{\partial z}\right)^{2}}{\frac{1}{K_{z}}\left(\frac{\partial \psi}{\partial r}\right)^{2}+\frac{1}{K_{r}}\left(\frac{\partial \psi}{\partial z}\right)^{2}}
$$

The following boundary conditions are used:

1) at the injection and extraction chambers: $q_{r}=-K_{r} \frac{\partial \varphi}{\partial r}$, with $q_{r}>0$ and $q_{r}<0$ respectively

2) $q_{r}$ or $q_{z}=0$ at no flow boundaries

3) $\phi=$ const on Dirichlet boundaries Submit your manuscript electronically for review.

\section{B. Transport Equation}

The reactive transport equation can be written as:

$$
R \frac{\partial C}{\partial t}=\frac{\partial}{\partial \zeta_{i}}\left(D_{i j} \frac{\partial C}{\partial \zeta_{j}}\right)-V_{i} \frac{\partial C}{\partial \zeta_{i}}-\lambda C+R_{m}
$$

where $C$ is tracer concentration [M/L3], $R$ is the retardation factor [-], $D$ is the dispersion coefficient $[L 2 / T], V$ is the pore water velocity in the streamline direction $[L / T]$, is the distance along the streamline direction and the perpendicular to it, represented by $i=1$ and 2 respectively $[L], t$ is time $[T], \lambda$ is the first order decay rate [T-1], and $R_{m}$ is the Monod sink/source term given by:

$$
R_{m}=-M_{t} V_{\max } \frac{C}{K_{s}+C}
$$


where $M_{t}$ is the biomass concentration, $V_{\max }(T-1)$ is the maximum rate of consumption of substrate (biodegradable tracer) and $\mathrm{Ks}$ is the half saturation rate constant [M/L3]. The biomass growth rate can be expressed as:

$$
\frac{\partial M_{t}}{\partial t}=\left(u_{\max } \frac{C}{K_{s}+C}-d\right) M_{t}
$$

where $u_{\max }$ is maximum specific growth rate $(T-1)$ and $\mathrm{d}$ is the specific decay rate for the microbial population. The change in substrate concentration due to microbial activity can be expressed as:

$$
\frac{\partial C}{\partial t}=-M_{t} V_{\max } \frac{C}{K_{s}+C}
$$

A time operator split technique is used separate the linear and non-linear terms in the reactive transport equation (4). First the linear transport equation including is solved linear sorption, linear decay. The newly computed concentrations are then used to solve the Monod equations (6) and (7) and evaluate a new $R_{m}$. This $R_{m}$ value is then replaced into equation (4) and a new concentration after the transport step is calculated. This iterative procedure is repeated until changes in concentration after a non-linear step and a transport step in small before proceeding to the next time step.

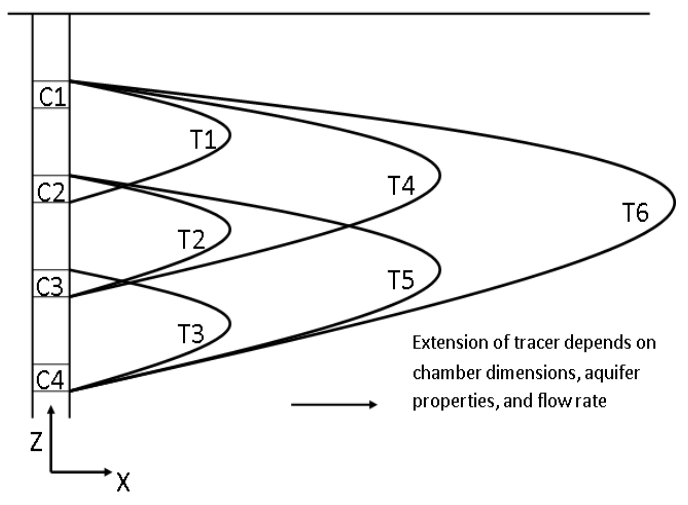

Fig. 1. Assembly of the MFRTT.

\section{MFRTT SETUP}

MFRTT consists of 4 separated chambers (C1, C2, C3, and C4, Fig. 2) each of which is divided into 4 sub-chambers. Using these 16 sub-chambers, 24 different tracer tests could be performed to characterize contaminant source zone in 4 directions. Fig. 1 shows vertical view of the MFRTT. This vertical view shows the outer stream line of 6 tracer tests performed in one of the four directions. For example T1, T2, and $\mathrm{T} 3$ are used to characterize aquifer volumes contained within outer streamlines of these three tests respectively. Then, T1, T2, and T4 are used to characterize aquifer volume contained within outer streamline of $\mathrm{T} 4$ and not contained in T1 or T2. Using different combinations of MFRTT BTCs, accurate characterization of contaminant source zone properties could be achieved. The extension of tracer in $x$ and $\theta$ directions depends on the chamber geometry, aquifer properties, and flow rate. The tracer tests can be used to evaluate the spatial patterns of contaminants in the source zone before and after implementing some in-situ technique for site cleanup, in order to evaluate the effectiveness of remediation achieved and the possible impacts of the cleanup technology on hydrodynamic and bio-geochemical processes.

\section{RESUlTS AND DISCUSSION}

Several example problems are simulated to examine the developed model and to present its capabilities. Unless otherwise noted, the results presented here are based on the dipole configuration and aquifer properties presented in Table I.

TABLE I: CONFIGURATION AND AQUIFER PROPERTIES FOR THE TESTING

\begin{tabular}{|l|l|}
\multicolumn{2}{l}{ PROBLEM } \\
\hline Dipole Configuration: & Aquifer Properties: \\
\hline$L=0.200 \mathrm{~m}(20 \mathrm{~cm})$ & $K_{r}=1 \times 10^{-5} \mathrm{~m} / \mathrm{s}$ \\
\hline$\Delta=0.050 \mathrm{~m}(5 \mathrm{~cm})$ & $K_{z}=1 \times 10^{-5} \mathrm{~m} / \mathrm{s}$ \\
\hline $\mathrm{r}_{w}=0.010 \mathrm{~m}(1 \mathrm{~cm})$ & $\phi=0.33$ \\
\hline$Q=6.667 \times 10-7 \mathrm{~m}^{3} / \mathrm{s}(40 \mathrm{~mL} / \mathrm{min})$ & \\
\hline
\end{tabular}

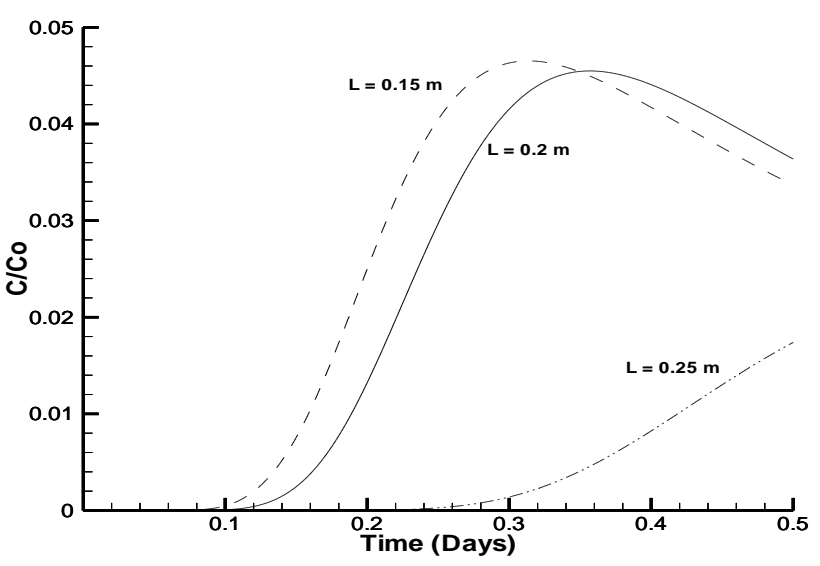

Fig. 2. Effect of changing $L$ on the breakthrough curve.

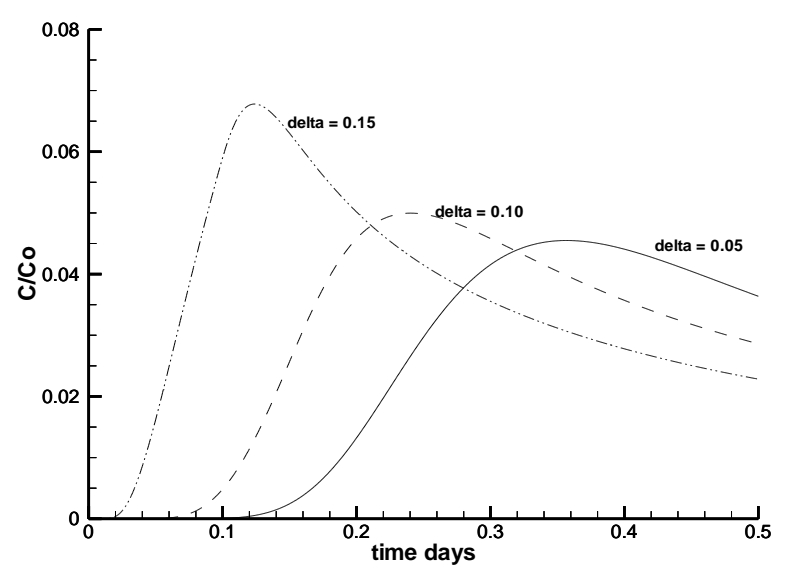

Fig. 3. Effect of changing $\Delta$ on the breakthrough curve.

Fig. 2 presents the tracer's breakthrough curve obtained at the extraction chamber for several values of $L$. As expected, the longer the shoulder length the longer it takes the tracer to arrive in the extraction chamber. Increasing $L$ from $0.2 \mathrm{~m}$ to $0.25 \mathrm{~m}$ may have caused losing some amount of the tracer in the aquifer which is reflected in the late appearance of the 
tracer ( 0.26 days) in the extraction chamber compared to the increase of $L$ from $0.15 \mathrm{~m}$ to $0.2 \mathrm{~m}$ when the tracer appeared after 0.12 days.

Fig. 3 shows the effect of changing $\Delta$ on the breakthrough curves for constant $L$. It is evident from this figure that tracer arrives faster to the extraction chamber with the increase of $\Delta$. That is because increasing $\Delta$, for a constant $L$, shortens the distance between the two chambers.

Fig. 4 demonstrates that increasing the flow between the injection and extraction chambers forces the tracer to travel faster and, therefore, show up earlier in the extraction chamber. Increasing $Q$ also ensures that most of the injected tracer is collected at the extraction chamber.

Fig. 5 illustrates the influence of the isotropy ratio $a^{2}$ on the breakthrough curve. Increasing this ratio (which means increasing $K_{r}$ ) eases the flow, and therefore the tracer transport, in the radial direction compared to the vertical one. This causes the tracer to take longer path before arriving in the extraction chamber. Consequently, some loss of the tracer in the aquifer is expected. Both effects are clearly shown in Fig. 4 when $a^{2}$ is increased from 0.5 to 1 and then to 2 .

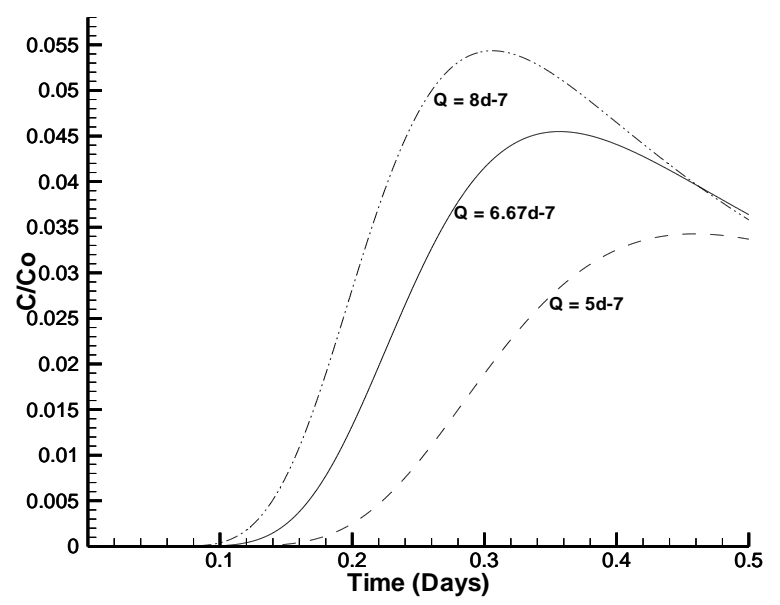

Fig. 4. Effect of changing $Q$ on the breakthrough curve.

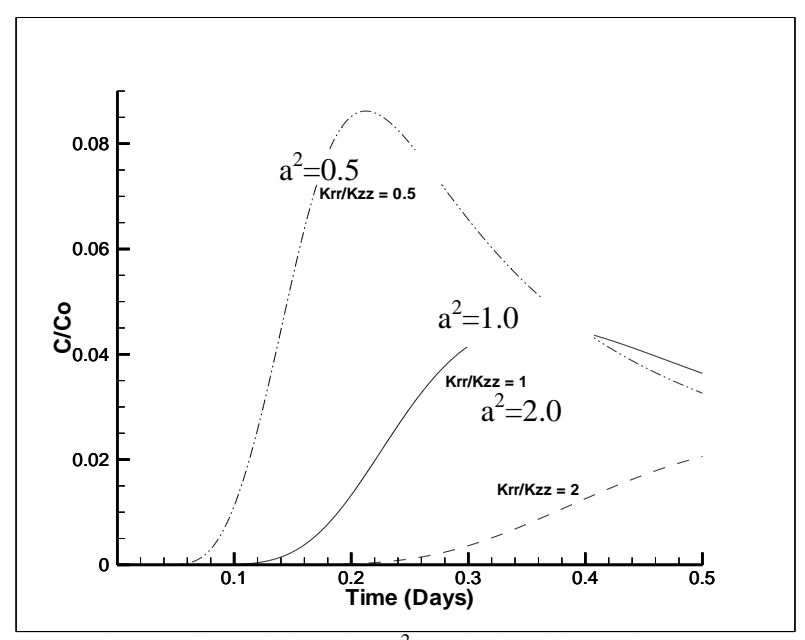

Fig. 5. Effect of changing $a^{2}$ on the breakthrough curve.

Fig. 6 shows the effect of increasing the retardation factor on the breakthrough curves. It is well known that increasing the retardation factor delays the travel of a tracer or contaminant. This is evident in this figure as only 0.3 days were enough for the inert tracer concentration to reach maximum value. On the other hand, 0.5 days were not enough time for the concentration to reach maximum in the other two cases when $R$ is increased to 2 and 3 respectively.

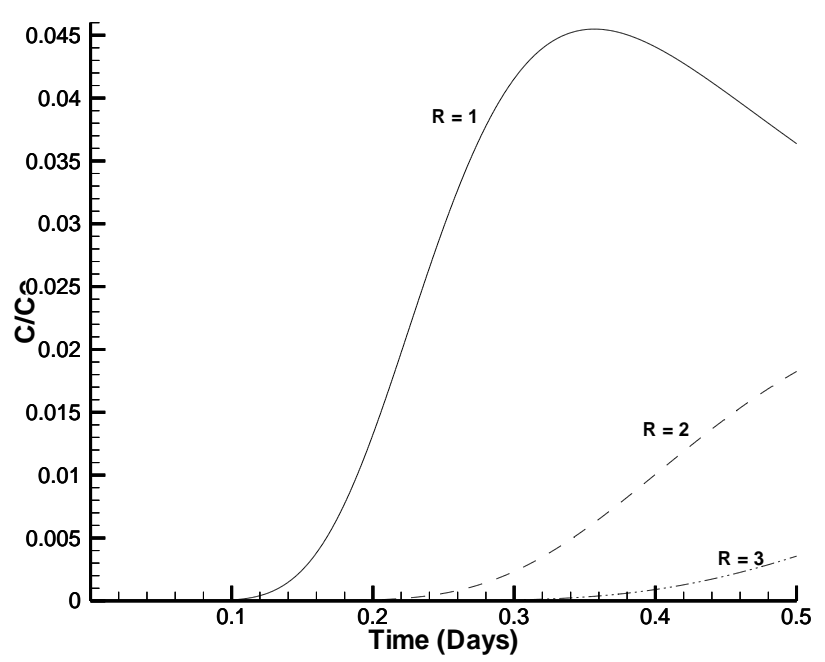

Fig. 6. Effect of changing $R$ on the breakthrough curve.

\section{CONCLUSIONS}

A numerical model which solves the radial saturated symmetric non-homogenous isotopic stream function equation was developed. This model accounts for hydraulic conductivity, and porosity; provides options for the location of upper and lower horizontal boundaries; allows for a user specified location of a horizontal feature with a thickness, hydraulic conductivity, and porosity; and accounts for an asymmetric dipole system. The DFRTT model was validated through comparisons with existing analytical solutions. Validation process proved model accuracy. Validation of the MFRTT is currently underway.

\section{ACKNOWLEDGEMENTS}

This work has been funded by the United Arab Emirates University and is an extension of a research project that was conducted at the University of Sheffield, UK.

\section{REFERENCES}

[1] Z. J. Kabala, "The dipole flow test: A new single-borehole test for aquifer characterization," Water Resour. Res., vol. 29, no. 1, pp. 99-107, 1993.

[2] M. Mohamed, D. Nayagum, N. Thomson, D. McKnight, C. Berryman, and S. Banwart, "The dipole flow and reactive tracer test: Initial field and modeling results," in Proc. the MODFLOW and More 2006: Managing Ground Water Systems, Poeter et al., Eds. Colorado, USA, 22-24 May, 2006, pp. 205-210.

[3] N. R. Thomson, G. Roos, R. D. Wilson, S. F. Thornton, and B. A Tolson, "The dipole flow and reactive tracer test for aquifer parameter estimation," presented at the AGU Fall Meeting, San Francisco, CA, Dec. 15-19, 2008.

[4] M. Mohamed, N. Thomson, and S. Banwart, "Interpretation of the dipole flow and reactive tracer test field parameters using PEST," in Proc. the Fifth MODELCARE International Conference on Calibration and Reliability in Groundwater Modelling, From Uncertainty to Decision, The Hague, Netherlands, 6-9 June 2005.

[5] B. Herrling and J. Stamm, "Numerical results of calculated 3D vertical circulation flows around wells with two screen sections for in situ or on-site aquifer remediation," Computational Methods in Water Resources IX, 1992.

[6] R. D. Philip and G. R. Walter, "Prediction of flow and hydraulic head fields from vertical circulation wells," Ground Water, vol. 30, no. 5, pp. 765-773, 1992. 
[7] V. A. Zlotnik and G. Ledder, "Effect of boundary conditions on dipole flow," in Computational Methods in Water Resources X, A. Peters et al., Ed. Norwell Mass., Kluwer Acad., 1994, vol. 2, pp. 907-914.

[8] V. A. Zlotnik and B. R. Zurbuchen, "Dipole probe: Design and field applications of a single-borehole device for measurements of vertical variations in hydraulic conductivity," Ground Water, vol. 36, no. 6, pp 884-893, 1998

[9] V. A. Zlotnik, B. R. Zurbuchen, and T. Prak, "The steady-state dipole-flow test for characterization of hydraulic conductivity statistics in a highly permeable aquifer: Horkheimer Insel Site, Germany," Ground Water, vol. 39, no. 4, pp. 504-516, 2001.

[10] T. Halihan and V. A. Zlotnik, "Asymmetric dipole-flow test in a fractured carbonate aquifer," Ground Water, vol. 40, no. 5, pp 491-499, 2002

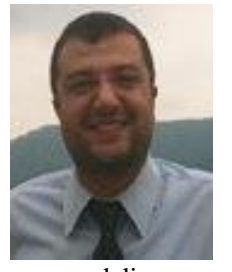

Mohamed Mostafa Mohamed is an associate professor of water resources and environmental engineering in the Department of Civil and Environmental Engineering at the United Arab Emirates University (UAEU). He received his B.Sc. and M.Sc. degrees from Cairo University, Egypt. Dr. Mohamed obtained his Ph.D. degree from the

University of Florida, USA, where his research focused on modeling contaminants transport and biotransformation in the subsurface. After serving for a year as a postdoctoral research associate at the University of Florida, and prior to his affiliation with the UAEU, he joined the GPRG (groundwater protection and restoration group) at Sheffield University, UK as a research scientist. Dr. Mohamed is a member of several international professional associations. He teaches several courses in the fields of water resources and environmental engineering. His research activities are focused on contaminants fate and transport in the subsurface groundwater bioremediation, stochastic subsurface hydrology, and in-situ characterization of contaminated groundwater sites. 